\title{
Learning to be Lazy: Exploiting Redundancy in a Novel Task to Minimize Movement-Related Effort
}

\author{
Rajiv Ranganathan, ${ }^{1,2}$ Adenike Adewuyi, ${ }^{4}$ and Ferdinando A. Mussa-Ivaldi ${ }^{1,2,3,4}$ \\ ${ }^{1}$ Sensory Motor Performance Program, Rehabilitation Institute of Chicago, Chicago, Illinois 60611, Departments of ${ }^{2}$ Physical Medicine and Rehabilitation \\ and ${ }^{3}$ Physiology, Northwestern University, Chicago, Illinois 60611, and ${ }^{4}$ Department of Biomedical Engineering, Northwestern University, Evanston, \\ Illinois 60208
}

\begin{abstract}
A key issue in motor control is to understand how the motor system chooses a solution from the multiple solutions that exist to achieve any particular task goal. One hypothesis is that redundancy may be resolved by minimizing movement-related costs. However, testing this prediction in motor learning has been problematic in simple laboratory tasks, like reaching, because the motor system already has extensive prior knowledge about redundancy in these tasks. Here, we used a novel task where healthy human participants performed finger movements to guide a computer cursor to different targets on the screen. Through training, all participants learned to perform successful goal-directed movements. Our findings showed that subjects did not develop a single inverse map from target to hand posture. Instead, they learned to use distinct hand postures to get to a single target, using a strategy in which the final hand posture at the target depended on the starting hand posture. Furthermore, postures chosen also depended upon the information content of visual feedback, with precise visual feedback resulting in postures that minimized movement-related costs. These results reinforce the idea that redundancy is exploited to minimize movement-related costs and that feedback plays a critical role in modulating this ability to effectively take advantage of the abundance of degrees of freedom.
\end{abstract}

\section{Introduction}

There exist two competing approaches in motor control that describe how the nervous system resolves the issue of redundancy (Bernstein, 1967). The first approach is to reduce redundancy by introducing constraints between the multiple degrees of freedom (Donders, 1848; Borghese et al., 1996), whereas the second approach resolves redundancy by minimizing movement-related costs (Hollerbach and Suh, 1987; Mussa-Ivaldi et al., 1988; Mussa-Ivaldi and Hogan, 1991; Todorov and Jordan, 2002). This latter strategy can be considered as "exploiting redundancy" because to achieve any particular task goal, the solution corresponding to the least movement cost is not unique-rather, different solutions to achieve the same task goal may be adopted depending on the initial conditions of the movement. In humans, evidence for exploiting redundancy to minimize movementrelated costs has been shown in several reaching studies where the choice of arm posture used to get the hand to a particular location in external space depends on the starting arm posture (Soechting et al., 1995; Gielen et al., 1997; Medendorp et al., 2000; Vetter et al., 2002).

\footnotetext{
Received March 24, 2012; revised Sept. 28, 2012; accepted 0ct. 3, 2012.

Author contributions: R.R., A.A., and F.A.M.-I. designed research; R.R. and A.A. performed research; F.A.M.-I. contributed unpublished reagents/analytic tools; R.R. analyzed data; R.R. and F.A.M.-I. wrote the paper.

This project was supported by a grant from the National Institutes of Health (1R01NS053581-01A2). We thank

Drs. Maura Casadio and Robert Scheidt for insightful comments on an earlier version of the manuscript.

The authors declare no competing financial interests.

Correspondence should be addressed to Rajiv Ranganathan, Robotics Lab, Rehabilitation Institute of Chicago,

345 E. Superior Street, Suite 1385, Chicago, IL 60611. E-mail: rrangana@northwestern.edu.

DOI:10.1523/JNEUROSCI.1553-12.2013

Copyright $\odot 2013$ the authors $\quad 0270-6474 / 13 / 332754-07 \$ 15.00 / 0$
}

This hypothesis that the nervous system resolves redundancy by minimizing movement-related costs makes a specific prediction in motor learning: early in learning, when the controller does not have adequate knowledge of the redundancy in the system, the final postures used for getting to a particular task location when starting from different starting postures should be less separable (i.e., less distinct). With further practice, however, once the controller determines the redundancy in the system, it could use this redundancy to minimize movement related-costs. This strategy would imply that the final postures chosen for a particular task location would be distinct for movements initiated from different starting postures, leading to an increase in separability with learning (along with a decrease in the movement-related cost). This prediction is in contrast to the constraint-inducing strategy where the separability between these final postures should decrease with learning (i.e., participants would effectively converge to a single final posture for getting to a task location since the redundancy is reduced by the induced constraints).

However, a potential difficulty with testing this prediction using familiar tasks like reaching is that the motor system already has extensive knowledge of how the multiple degrees of freedom relate to the task-relevant variable (e.g., how changes in the joint angles of the arm relate to changes in hand position) and therefore, this prior knowledge of redundancy may confound the effects of learning in these tasks. Here, we addressed this issue by using a novel task which involved controlling a computer cursor using changes in hand posture (Mosier et al., 2005; Danziger et al., 2009; Liu et al., 2011). We examined the hand posture adopted when the cursor was always at a single target location. We found that participants adopted distinct hand postures at the 
A
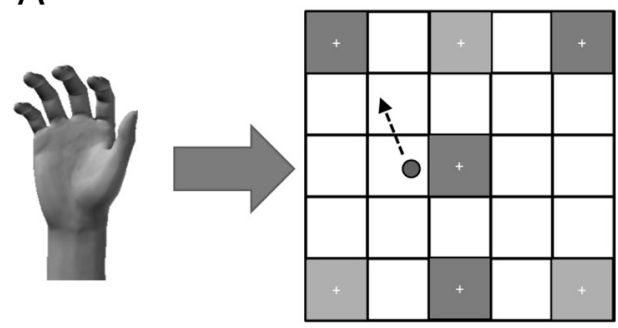

$h_{19 \times 1}$
B

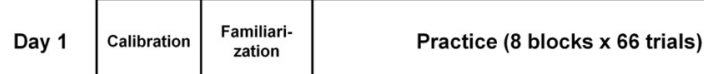

Day 2
Practice ( 8 blocks $\times 66$ trials)
$\mathrm{C}$

\section{Precise}

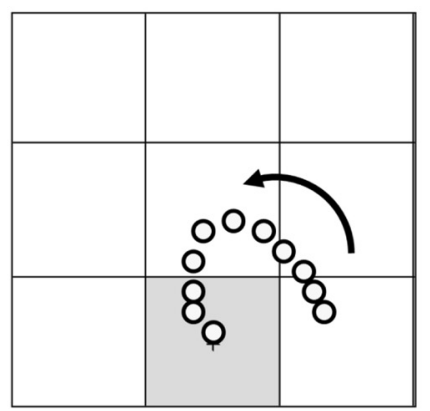

Intermittent

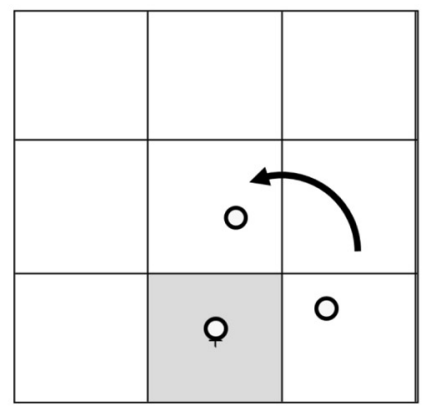

Blur

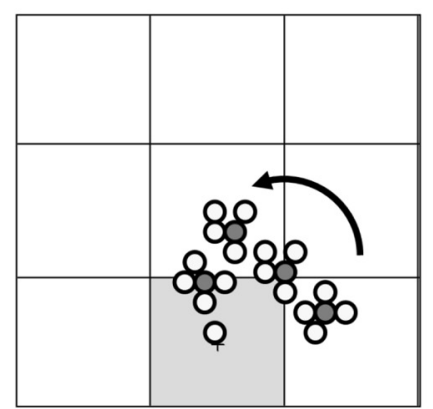

Figure 1. Experimental setup. $A$, Schematic of the task. Participants wore a Cyberglove that measured 19 degrees of freedom from the hand. This 19-dimensional vector (h) was linearly mapped to the 2-D position (p) of a cursor on a screen. Participants were instructed to move the cursor to different targets shown on the screen —-the dark gray targets were the training targets and the light gray targets were the generalization targets. B, Practice schedule for participants on Day 1 and Day 2. C, Visual feedback for different groups for a movement performed in the direction indicated by the arrow. For the purpose of clarity, only a subset of the $5 \times 5$ target grid is shown. At any point in time, all groups saw only the instantaneous position of the cursor (i.e., no information about the previous positions was provided).

target, using a strategy in which the final hand posture depended on the starting hand posture. This strategy tended to minimize movement-related costs, but only in the presence of precise visual feedback.

\section{Materials and Methods}

\section{Participants}

Eighteen young healthy adults ( 9 females, mean age $=24 \pm 2$ years) participated in the study. All participants were right handed. Participants provided informed consent and all procedures were approved by the Northwestern University IRB.

\section{Task}

Participants wore a Cyberglove (Immersion Technologies) on their right hand that measured a total of 19 joint angles (the 3 wrist sensors were excluded). The 19 joint angles of the hand were linearly mapped on to the position of a cursor on a screen and the task of the participants was to move the cursor to different targets that were displayed on a $5 \times 5$ grid (Fig. 1A). The sampling frequency was set at $60 \mathrm{~Hz}$. Vision of the participants' arm and hand was obscured using black cloth that was draped over the arm.

The 19-dimensional vector (h) representing the hand posture was linearly mapped on to the 2-D position of the cursor (p) using the equation below (Mosier et al., 2005; Liu and Scheidt, 2008):

$\left[\begin{array}{l}x \\ y\end{array}\right]=\left[\begin{array}{lllll}a_{1,1} & a_{1,2} & \cdots & a_{1,18} & a_{1,19} \\ a_{2,1} & a_{2,2} & & a_{2,18} & a_{2,19}\end{array}\right] \times$

$$
\left[\begin{array}{lllll}
h_{1} & h_{2} & \ldots & h_{18} & h_{19}
\end{array}\right]^{T}+\left[\begin{array}{l}
x_{0} \\
y_{0}
\end{array}\right]
$$

i.e., $\mathbf{p}=\mathbf{A h}+\mathbf{p}_{0}$

where the values for the mapping matrix $(\mathbf{A})$ and the offset $\left(\mathbf{p}_{0}\right)$ were determined from an initial calibration phase. Throughout the manuscript, vectors are represented by characters in lower case bold face whereas matrices are represented by characters in upper case bold face.
This mapping meant that each hand posture was mapped to a single cursor location but a single cursor location could be achieved using multiple hand postures. Therefore, to guide the cursor to different $(x, y)$ locations on the screen, participants had to perform finger movements to change from one hand posture to another. Holding a static hand posture resulted in the cursor staying at a single $(x, y)$ location.

Procedure

A schematic of the procedures is shown in Figure $1 \mathrm{~B}$. There were three main phases in the experiment-calibration, familiarization and training.

Calibration. To determine the mapping matrix $\mathbf{A}$ and the offset $\mathbf{p}_{\mathbf{0}}$, we had participants perform a sequence of free finger movements where they were instructed to move their fingers in as many different ways as possible while avoiding extremes of range of motion (Casadio et al., 2010 ) until we obtained $4000-5000$ samples ( $~ 70-90$ s). Visual feedback of the 19 individual sensors was provided as a bar graph to encourage participants to explore different movements. Principal component analysis (Daffertshofer et al., 2004) using the covariance matrix was performed on this dataset and the first 2 principal components (PCs) were used as the two rows of the A matrix (i.e., the first PC corresponded to motion along the $x$-axis and the second PC to motion along the $y$-axis). Because the variation along the first $\mathrm{PC}$ is always greater than or equal to that along the second PC (by definition), the PCs were scaled by the square root of the respective eigenvalue to make the task difficulty comparable along both directions. The offset $\mathbf{p}_{\mathbf{0}}$ was computed so that the mean posture from the calibration phase corresponded to the center of the $5 \times 5$ grid.

Familiarization. Before training, participants performed a familiarization phase that involved trying to reach all 25 targets of the $5 \times 5$ grid. This was done just to ensure that all targets in the workspace were reachable. All participants were able to reach the targets without requiring a rescaling of the original mapping matrix.

Training. Following the familiarization block, participants practiced on 4 targets - 3 outer targets and one center target (indicated in dark gray 
squares in Fig. $1 \mathrm{~A}$ ). Practice consisted of 16 blocks that were spread over $2 \mathrm{~d}$ into 8 blocks/d. Each block consisted of 66 movements (60 movements with visual feedback, 3 "blind" catch trials and 3 return movements following the catch trials with visual feedback). The sequence of targets in each block was randomized but always consisted of 24 movements to the center target ( 8 from each outer target) and 12 movements to each of the 3 outer targets. The choice of this sequence was used as a trade-off between maximizing the number of movements to the center target (which was the focus of the data analysis) and minimizing the total number of movements (to avoid excessive fatigue within a single practice session). Participants were instructed to get to the center of each target within a movement time of $2 \mathrm{~s}$.

Training groups. Participants were randomly assigned into one of three groups that differed in the quality of visual feedback during practice (Fig. 1C). (A) In the Precise group, participants were given precise spatial information and continuous temporal feedback of the cursor position which was displayed as small circle. (B) In the Intermittent group, participants were provided precise spatial position of the cursor, but were shown the cursor only intermittently in time. The cursor was displayed only for $0.1 \mathrm{~s}$ every $0.5 \mathrm{~s}$. (C) In the Blur group, participants were given continuous temporal feedback, but the spatial position of the cursor was corrupted with white Gaussian noise with zero mean and SD 0.2 (units were normalized relative to the size of the target square). This resulted in participants seeing a cloud of 4-6 cursors around the actual position of the cursor (Stevenson et al., 2009). All feedback manipulations were present only during movement. It is important to note that in all cases, participants saw only the instantaneous position of the cursor (i.e., the trail of cursors along the path taken by the cursor was not actually seen by the participant and is shown in the figure only for clarity). Four out-ofbounds indicators (up, down, left, right) surrounding the $5 \times 5$ grid were used to give feedback when participants moved outside the grid in the corresponding direction.

When the cursor stopped inside the target, precise visual feedback was provided for all groups. At the end of each trial, the corresponding target lighted up in yellow if the participants were inside the target within a movement time of $2 \mathrm{~s}$. If participants took longer than $2 \mathrm{~s}$ to reach the target, the target was highlighted in red. A scoring system based on the movement time and accuracy was also used to keep participants motivated. The next target in the sequence was presented only when the participant successfully reached the previous target.

Blind trials. In each training block, there were 3 blind trials that appeared randomly, but with the constraint that no blind trials appeared in the first 30 movements. Visual feedback of the cursor was unexpectedly removed during these trials. The blind trials were always directed from the center target to the outer targets. At the end of the catch trial, the cursor position was given as feedback and was subsequently followed by a return movement back to the center target. During the first 12 blocks of practice, the blind trials were directed to the same targets as those being trained, whereas in the last 4 blocks of practice the blind trials (i.e., at the end of Day 2) were directed to three generalization targets that were not part of the training targets (indicated by the light gray squares in Fig. $1 \mathrm{~A}$ ).

\section{Data analysis}

To simplify statistical analysis and presentation of the data, we grouped the 8 practice blocks of each day into two phases-the early phase, which consisted of the first 4 blocks, and the late phase, which consisted of the last 4 blocks. This meant that each phase consisted of 96 postures at the center target ( 32 from each outer target), which also provides greater robustness for the estimates of variance and classification accuracy.

Task performance. The task performance was quantified by two metrics - the Euclidean error and movement straightness. The Euclidean error was quantified by the Euclidean distance between the center of the target and the cursor at the end of the movement. The end of the movement was defined as the point at which the distance between two successive glove data samples did not change by $>2$ units $\left(\sim 1^{\circ}\right)$ for 10 consecutive samples. If the movement time was $>2 \mathrm{~s}$, the error was computed at $2 \mathrm{~s}$ into the movement. For the blind trials with no visual feedback, the error was computed at the end of movement. Movement straightness was measured using the aspect ratio, i.e., the maximum perpendicular distance on the screen (measured from the line joining the start and the end points of the movement) normalized to the straight line distance between the start and end points.

Variance accounted for by first 2 PCs. The hand postures adopted throughout the whole movement in each phase were analyzed using principal component analysis. Since the task could theoretically be achieved by using only 2 PCs (the task space is planar), we examined the fraction of variance accounted for by 2 PCs and how this changed with learning. We eliminated postures when the hand was static (i.e., when there was no change in the glove data) so that these postures were not overrepresented in the data.

Null space-task space variability at the center target. For movements to the center target, the final postures adopted at rest were separated into null-space and the task-space components, i.e., $\mathbf{h}=\mathbf{h}_{\mathbf{n}}+\mathbf{h}_{\mathbf{t}}$, where $\mathbf{h}_{\mathbf{n}}=$ $\left(\mathbf{I}_{19}-\mathbf{A}^{+} \mathbf{A}\right) \mathbf{h}$ and $\mathbf{h}_{\mathbf{t}}=\left(\mathbf{A}^{+} \mathbf{A}\right) \mathbf{h}$. $\mathbf{I}_{19}$ represents the $19 \times 19$ identity matrix and $\mathbf{A}^{+}$represents the Moore-Penrose pseudoinverse of the mapping matrix A (Mosier et al., 2005; Liu and Scheidt, 2008). As mentioned earlier, we chose only the center target for the analysis because the biomechanical constraints were minimized at this target. The center target corresponded to the mean hand posture during calibration, where all the joints were close to the middle of their range of motion. The total variance of these postures was then computed separately in the null space and the task space. Since the two spaces have different dimensions, the variances were normalized by the number of degrees of freedom in the task space and the null space (Scholz and Schöner, 1999).

Separability of postures at the center target. To determine whether postures at the center target (in either the null space or the task space) were separable by the starting target, we used linear discriminant analysis. The postures in the null space and task space were computed by directly projecting the hand posture on to the $17-\mathrm{D}$ null space or the 2-D task space (unlike the calculation for the variance, the null space and task space could not be embedded in the 19-D space since the linear discriminant analysis requires a full rank covariance matrix). We used half of the dataset to train the classifier and then cross-validated the classification by testing the classifier's ability to predict the starting target for the remaining half of the postures. Confidence in this procedure was estimated using a bootstrapping procedure (i.e., by repeating 50 times and randomly selecting $50 \%$ the postures for the training dataset each time). The mean classification accuracy of these 50 iterations was used as the index of separability between the 3 clusters representing postures at the center target that originated from the three outer targets). A mean classification accuracy of $100 \%$ indicates completely separable postures whereas $33 \%$ indicates no separation (chance level).

Transport efficiency. If the separability metric showed that there were redundant solutions, we also examined whether the null space was being "effectively used" using a transport efficiency metric. To determine the transport efficiency (i.e., how much was the movement-related effort in moving to the center target), we used a surrogate analysis and computed the movement cost for the solutions that were actually used and compared it to movement costs for other possible solutions that could have potentially been used (similar to the randomization method used by Müller and Sternad, 2003). In other words, if the mean posture used for the outer targets is represented by $T_{i}$ and the corresponding posture at the center target was $C_{i}$, we compared the actual movement cost (i.e., from $T_{i}$ to $C_{i}$ ) to the cost of alternative possible solutions that could have been used (i.e., from $T_{i}$ to $C_{j}$ where $i \neq j$ ) (see Fig. $4 A$ ). Although some studies have used formal definitions of movement cost (such as mechanical workSoechting et al., 1995), given the significant complexity in the hand due to the high number of degrees of freedom and the shared musculature, we chose to use a simpler metric to quantify the movement cost by using the Euclidean distance between two hand postures.

For each set of postures adopted (i.e., at the three outer targets and at the center target from each of these targets), a $3 \times 3$ Euclidean distance matrix was constructed-where each element $d_{i j}=\left\|\mathbf{t}_{\boldsymbol{i}}-\mathbf{c}_{j}\right\|$, where $\mathbf{t}_{\boldsymbol{i}}$ represents the mean posture at the $i$ th outer-target and $\mathbf{c}_{j}$ represents the actual mean center-target posture adopted when the movement started 


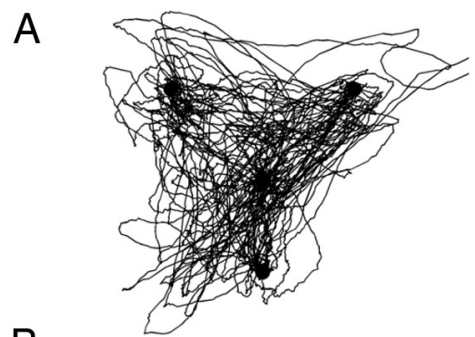

B
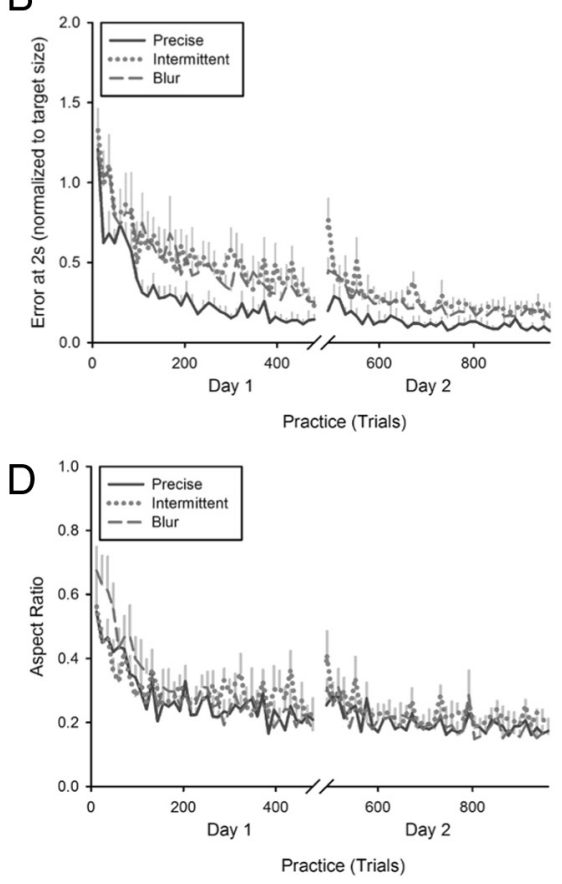
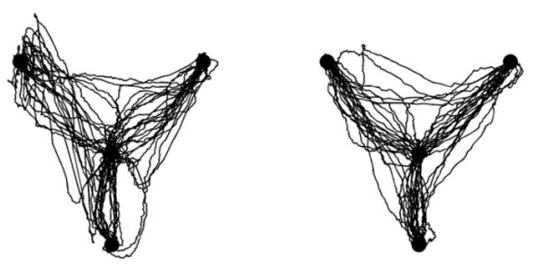

C

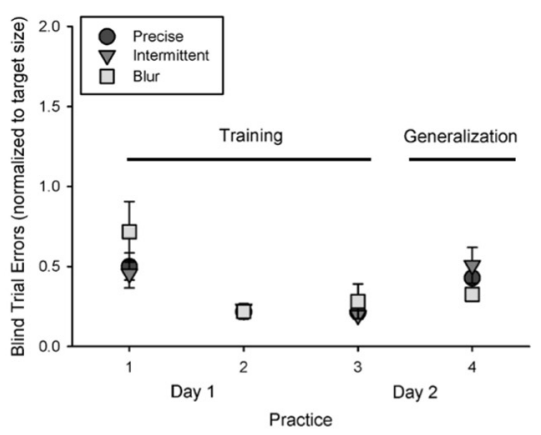

E

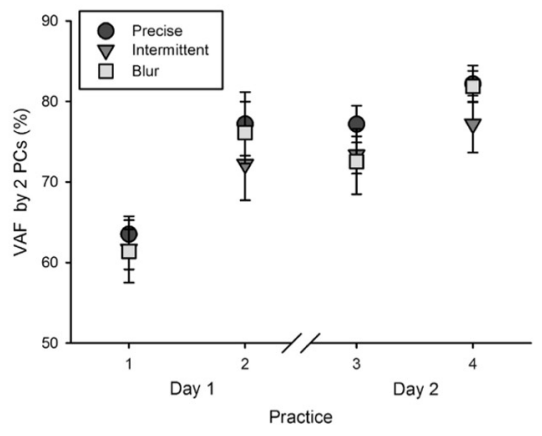

Figure 2. A, Sample data of cursor paths for trials with visual feedback for a single subject in the precise group at three different stages of practice: beginning (trials 1-60), intermediate (trials 181-240) and at the end (trials 901-960). B, C, Euclidean error computed at 2 sinto the movement during the training trials with visual feedback $(\boldsymbol{B})$ and in blind trials $(\boldsymbol{C})$. For the blind trials, the first three blocks (two on Day 1 and the first on Day 2) involved the 3 training targets whereas the fourth block was a test of generalization to 3 untrained targets. D, Movement straightness during practice for trials with visual feedback as quantified by the aspect ratio. $\boldsymbol{E}$, Variance accounted for (VAF) by the first 2 PCs during each practice block. Error bars represent 1 SE (between-participant).

from the $j$ th outer target. Once this distance matrix was determined, we computed the transport efficiency (TE) as

$$
\mathrm{TE}=\left(1-\frac{\sum_{i} d_{i, i} / 3}{\sum_{i \neq j} d_{i, j} / 6}\right) \cdot 100 .
$$

We bootstrapped this analysis 50 times by randomly selecting only half of the postures each time to estimate the mean and SD of the transport efficiency for each subject.

\section{Statistical analysis}

Statistical analyses on all dependent variables were performed using a Practice block (4) $\times$ Group (3) mixed-model ANOVA with practice block (1-4) as the within-subject factor and group (Precise, Intermittent, Blur) as the between-subject factor. When appropriate, corrections for sphericity were performed using the Greenhouse-Geisser correction. Post hoc comparisons for the practice block factor was done between the first and last block whereas for the group factor, the comparisons were done only between Precise and the other two groups using a Bonferroni correction. In the case of the transport efficiency where we were interested in the individual-subject data, we ran a one tailed $t$ test to examine whether the transport efficiency was significantly $>0$ for each subject. We also tested whether the mean transport efficiency of each group was

significantly greater than zero. The significance level of all tests was set at 0.05 . For the betweengroup comparisons, we only compared the Precise group with the Intermittent and the Blur groups.

\section{Results \\ Baseline}

To verify whether there were any differences between the groups at baseline, we compared the average time required to reach a target during the initial familiarization phase when all participants had precise visual feedback. The results showed that the differences between groups were not significant at baseline $\left.F_{(2,15)}=0.18, p=0.83\right)$. The mean $( \pm \mathrm{SD})$ movement times in the Precise, Intermittent and Blur groups were $9.7 \pm$ $4.0 \mathrm{~s}, 10.9 \pm 6.3 \mathrm{~s}$ and $9.2 \pm 4.2 \mathrm{~s}$ respectively.

\section{Euclidean error}

Sample cursor trajectories (only for trials with visual feedback) for one subject in the precise group at different stages of learning are shown (Fig. 2A). The analysis of the Euclidean error showed that participants learned to reduce their error with each practice block $\left(F_{(1.8,27.4)}=\right.$ 62.77, $p<0.01$ ), almost approaching a plateau by the last practice block (Fig. $2 B)$. Post hoc comparisons showed that the error in the last block of practice was significantly lower than the first block $(p<0.05)$. In addition, there was also a significant main effect of group, $F_{(2,15)}=5.79, p=0.014$. Post hoc comparisons showed that the error was lower in the precise group compared with both the blur and intermittent

groups $(p<0.05)$.

\section{Blind trials}

The analysis for the blind trials was performed separately for the three blocks that were toward the training targets, and the fourth block that had the generalization (untrained) targets (Fig. 2C). For the blind trials toward the training targets, there was a reduction in error for the blind trials with practice $\left(F_{(1.4,20.4)}=22.65\right.$, $p<0.01$ ), but no significant differences between groups. Post hoc comparisons showed that the error in the last block of practice was significantly lower than the first block $(p<0.05)$. The errors for the generalization targets tended to be lower than the initial blind trials at the trained targets (Fig. $2 B$ ) although this was not statistically significant $\left(F_{(1,15)}=2.79, p=0.11\right)$. There were no significant differences between the groups. These blind trials were not considered for further analysis.

\section{Aspect ratio}

Participants produced straighter paths of the cursor with practice $\left(F_{(1.9,27.9)}=47.37, p<0.01\right)$, again approaching a plateau by the late phase of Day 2 (Fig. 2D). Post hoc comparisons showed that 
the aspect ratio in the last block was significantly lower than the first block $(p<$ $0.05)$. There were no significant differences between the groups.

\section{Variance accounted for by 2 PCs}

The percentage of variance accounted for by the first 2 PCs increased with practice $\left(F_{(3,45)}=48.53, p<0.01\right)$, indicating that the hand postures used were closer to lying on a 2-D plane (Fig. 2E). Post hoc comparisons showed that the percentage of variance was significantly higher on the last block of practice compared with the first block $(p<0.05)$. No significant effect of group was found.

\section{Variance in null-space and task-space at the center target}

There was a significant reduction of both null space and task space variance with practice. For the null-space variance, there was a significant main effect of practice block $\left(F_{(3,45)}=22.95, p<0.01\right)$. Post hoc comparisons showed that the variance in the null space was smaller on the last block of practice compared with the first block $(p<0.05)$. The main effect of group was not significant (Fig. 3A).

For the task-space variance, there was a significant main effect of practice block $\left(F_{(1.8,26.5)}=37.58, p<0.01\right)$ and also a practice block $\times$ group interaction $\left(F_{(3.53,26.5)}=2.89, p=0.046\right)$. Analysis of this interaction showed that although all groups decreased their task space variance significantly with practice, the Intermittent group continued to decrease its variance significantly even on Day 2 (i.e., from blocks 3 and 4$)(p<0.05)$, whereas the other two groups did not show a significant decrease on Day 2 (Fig. 3B).

\section{Separability of postures at the center target}

The separability of postures in the null space increased with practice whereas the separability of postures in the task space showed no change. In the null space, the analysis showed a significant main effect of practice block $\left(F_{(3,45)}=21.96, p<0.01\right)$ and post hoc comparisons showed that the separability between postures in the null space was higher on the last block of practice compared with the first block $(p<0.05)$. The main effect of group was not significant (Fig. 3C). However, when considering the separability in the task space, the analysis showed no significant main effects or interactions (Fig. 3D).

\section{Transport efficiency}

When we compared the group differences in the early phase of Day 1, none of the groups showed a transport efficiency that was significantly greater than zero. However, when we compared the groups at the late phase on Day 2, only the precise group showed a transport efficiency that was significantly $>0$ $(p<0.05)$ (Fig. 4B). Analyses on individual participants
B Task Space

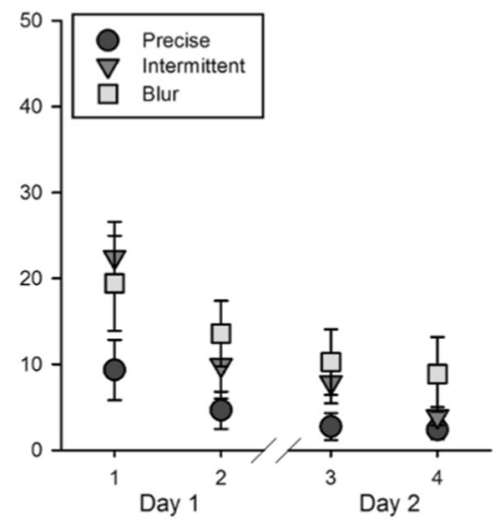

Practice

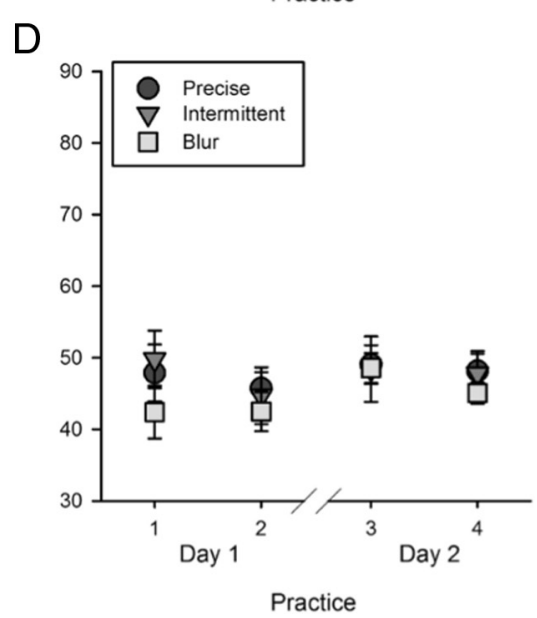

Figure 3. $\boldsymbol{A}, \boldsymbol{B}$, Variance of the postures at the center target in the null space $(\boldsymbol{A})$ and task space $(\boldsymbol{B})$ as a function of practice (normalized per d) for the three groups. The variance decreased in both the task space and the null space for all ps with practice. Note that the scales on the vertical axes are not equal. C, D, Classification accuracy for the different in space $(\boldsymbol{C})$ and task space $(\boldsymbol{D})$ as a function of practice. The classification accuracy was computed using a linear repeated 50 times for each subject and the mean classification accuracy was computed. Note that the classification accuracy in the null space increases with learning, whereas the classification accuracy in the task space does not change with learning. Error bars represent 1 SE (between-participant).

showed that all 6 subjects in the precise group showed transport efficiency significantly greater than zero $(p<0.05)$.

\section{Control experiment to check for glove hysteresis}

To rule out the possibility that the separability in postures was simply due to some form of hysteresis in the Cyberglove, we ran a control condition with a single subject. A custom-made plaster cast (which covered only the palm and not the dorsal part of the hand) was made with the subject's hand in a fixed posture with the MCP joints in slight flexion (similar to the posture of grasping a computer mouse). After the calibration phase to calculate the A matrix, the offset $\mathbf{p}_{\mathbf{0}}$ was adjusted so that resting the hand inside the cast would result in the cursor landing in the center target. This was done to effectively mimic the strategy of using the same hand posture to get to the center target. Four blocks of 60 trials were repeated with the only one difference: whenever the center target was presented, the participant simply placed his hand in the cast. When we classified the postures at the center target as a function of the starting posture, we found that the mean $( \pm S D)$ classification accuracy was $38 \pm 8 \%$ in the null space and $38 \pm 6 \%$ in the task 

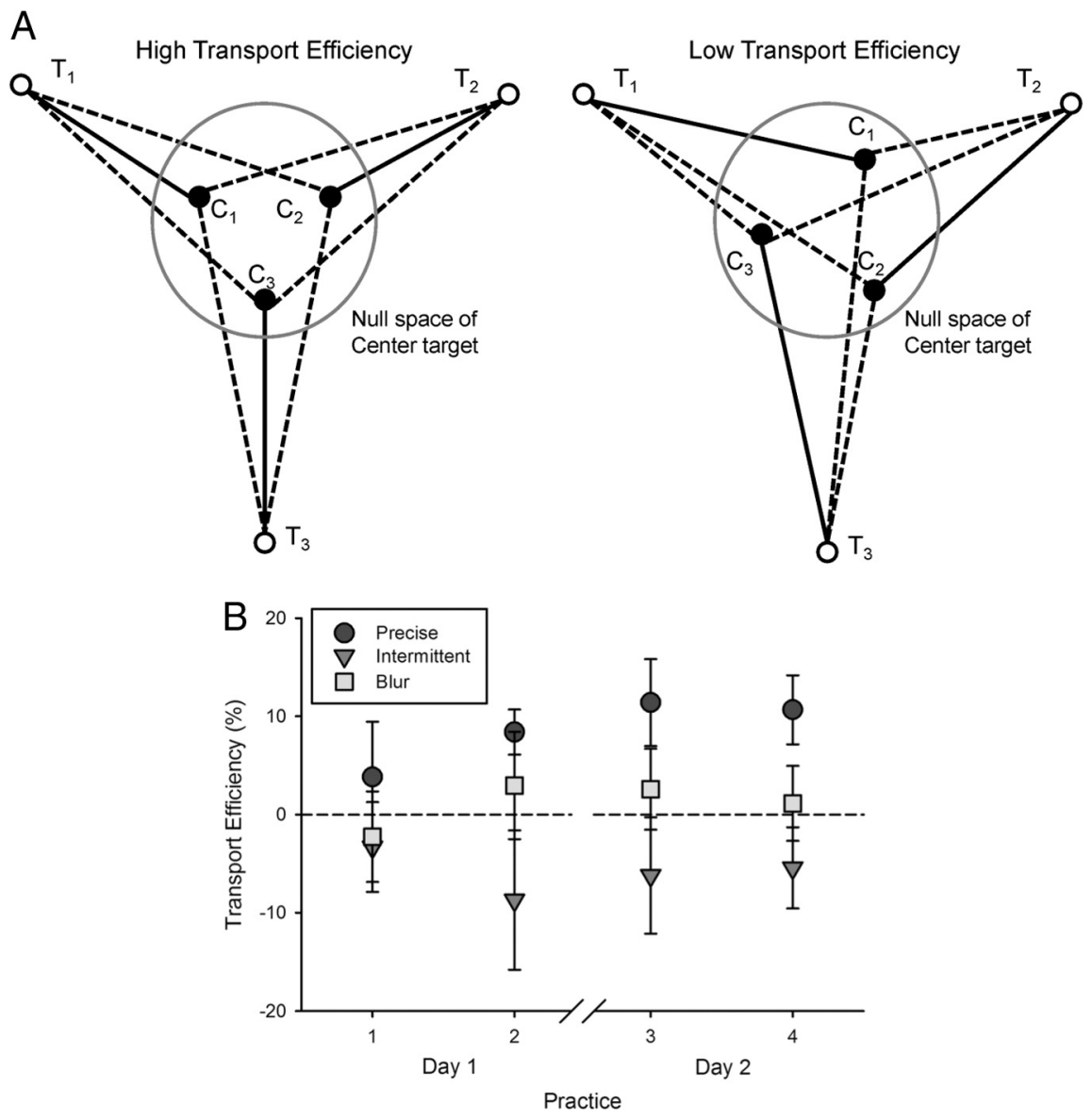

Figure 4. Schematic to illustrate how transport efficiency can be different when postures at the center are separable. The three postures at the outer targets are denoted by $T_{1}, T_{2}$, and $T_{3}$ and when the movement is initiated from these targets, the corresponding postures at the center target are $C_{1}, C_{2}$, and $C_{3}$. The transport efficiency effectively compares the actual movement costs used by the subject (represented by the length of the solid lines originating from the actual starting targets- e.g., $T_{1}-C_{1}, T_{2}-C_{2}$ etc.) to other possible movement costs (represented by the average length of the dotted lines originating from the other targets- e.g., $\mathrm{T}_{2}-C_{1}, T_{3}-C_{1}$ etc.). In the case of high transport efficiency $(\boldsymbol{A}$, Left), the solid lines are shorter than the average of the dotted lines, indicating that the actual costs observed were smaller than the costs for other alternative solutions. However in the case of low transport efficiency $(\boldsymbol{A}$, Right), the length of the solid lines are approximately equal to the average of the dotted lines, indicating that the actual cost was not much lower than other alternative solutions. It is important to note that even though $C_{1}, C_{2}, C_{3}$ are shown separated, they all represent postures at a single target location (i.e., the separation is only in the null space and not in the task space). $\boldsymbol{B}$, Transport efficiency for the different groups as a function of practice. Only the precise group showed transport efficiency significantly $>0$. Error bars represent $1 \mathrm{SE}$ (between-participant).

space. The low classification accuracy in these results show that the higher separability in the null-space observed in the participants during the experiment was not attributable to hysteresis in the glove.

\section{Discussion}

The aim of the experiment was to examine the role of redundancy when learning a novel task. Unlike tasks such as reaching where the motor system already has extensive experience with the redundancy in the motor system, here participants were completely naive to how the hand posture was mapped to the cursor position and had to discover this relation through practice. While previous studies using this protocol have examined overall changes in the null space and task space variability with learning, here we addressed the issue of whether examining the structure of this variability could yield more insight into how redundancy is used during learning.

The results of the experiment revealed two important findings: First, participants used distinct hand postures to get to the center target that systematically depended on the starting posture. The sep- arability between these hand postures at the center target increased with learning, indicating that participants were exploiting the redundancy in the task. Second, in the presence of precise visual feedback, there was clear evidence that the strategy of using distinct hand postures from different starting targets minimized movement-related costs. This suggested that participants were not learning a posture map (i.e., associating a cursor position to a hand posture), but a transport map (i.e., associating changes in cursor position to changes in hand posture).

Our finding that the strategy chosen was a transport map closely parallels the results from reaching studies (Soechting et al., 1995; Fischer et al., 1997; Vetter et al., 2002). However, unlike earlier studies that focused on well practiced tasks (where the nervous system likely has adequate knowledge of the redundancy in the body), the current results clearly show how this transport map is formed even in cases where such knowledge of the redundancy can only be acquired with practice. Initially, when the participants learned the map, they showed high variability in the postures they select to achieve the center target. With more practice, the variability of postures was reduced but the separability increased, indicating that learning did not cause convergence into a single posture. Critically, this separation between postures was only observed in the null space and not in the task space. In other words, using the cursor position at the center target alone was not sufficient to predict the starting target of the movement whereas the projection of the hand posture in the null space was able to discriminate the starting target with high accuracy. Further, the fact that the separability increased for all groups with practice indicated that this use of redundancy was not dependent upon visual feedback of the entire trajectory. These results are consistent with the predictions of the posture model from Rosenbaum et al. (1995) with the exception of the fact that although practice resulted in a reduction in variability of the postures, it did not result in a decreased influence of the start posture on the final posture.

In addition to finding that there was a use of the null space (i.e., using redundant solutions), the transport efficiency metric further revealed that the separation in null space was a strategy to reduce changes in hand posture, supporting the idea that the motor system effectively uses redundancy to minimize movement-related costs. Here, we found that the precision of visual feedback had a critical role in determining the optimality of the chosen postures. In particular, degrading the precision of visual feedback of the cursor when it was in motion either spatially (Blur) or temporally (Intermittent) not only resulted in slower rates of learning as seen in the Euclidean error and longer movement times (Stevenson et al., 2009), but also resulted in the choice of sub-optimal postures. Even though the separability was high in all groups, only the precise 
feedback group showed transport efficiency that was significantly greater than zero. Therefore, even though the visual feedback was only restricted to the task space, which allowed participants to minimize the Euclidean error from the center of the target, the visual information also provided them with a better estimate of which motions contribute to task space and null space motion. This allowed participants to minimize wasteful motions in the null space. We conclude that the ability to effectively use redundancy is critically mediated by feedback (Todorov and Jordan, 2002; Ranganathan and Newell, 2008, 2009). Furthermore, this finding also supports the idea that precise visual information of the entire trajectory of the cursor contributes to the learning of the map rather than simply the final position (Liu and Scheidt, 2008).

Finally, the results are also relevant to a recent debate over the role of the null space variability in motor learning. One hypothesis is that null space variability need not be decreased with learning because it is either irrelevant to task performance (Todorov and Jordan, 2002) or that it is actually "good variance" because it reflects the ability of the system to use multiple solutions (Latash et al., 2002). However, on the other hand, other studies have shown that there is a systematic decrease in null space variability with learning and hypothesize that this may be important for forming an inverse map that uniquely maps the lower dimensional task space to the higher dimensional body space (e.g., joint angles, forces; Mosier et al., 2005; Liu and Scheidt, 2008; Casadio et al., 2010; Liu et al., 2011). The current results show that both hypotheses are not mutually exclusive. There was clearly evidence of formation of an inverse map: with learning, postures occupied a largely 2-D plane (with 2 PCs accounting for $\sim 80 \%$ of the variance). Although some of this variance could be attributed to the influence of peripheral and central constraints on the hand (Schieber and Santello, 2004), the reduction in null space variability at a single target location (which could have theoretically been achieved using the same posture) shows that there was indeed an active reduction in null space variability. This suggests that participants were forming a stable inverse map by eliminating most of the null space variability. However, despite this reduction, participants were also able to use the remaining null space to minimize movement-related costs (i.e., minimizing changes in hand posture), showing that residual null space variability is not simply noise but rather, it retains structure that reflects control optimizations entirely orthogonal to the kinematic goals of the task. A similar approach to reconcile these seemingly contradictory notions of optimality and redundancy has also been recently described in force control tasks (Park et al., 2010).

In summary, we found that even in a novel task where there is no prior knowledge of how the degrees of freedom map to the task space, the nervous system learns to exploit redundancy to minimize movement-related costs, similar to arm movements (Soechting et al., 1995). However, feedback plays an important role since the movement costs are minimized only in the presence of precise visual feedback. Future experiments are needed to address whether extended learning at the task could result in efficient movements without reliance on visual feedback.

\section{References}

Bernstein NA (1967) The co-ordination and regulation of movements. Oxford: Pergamon.

Borghese NA, Bianchi L, Lacquaniti F (1996) Kinematic determinants of human locomotion. J Physiol 494:863-879. Medline

Casadio M, Pressman A, Fishbach A, Danziger Z, Acosta S, Chen D, Tseng HY, Mussa-Ivaldi FA (2010) Functional reorganization of upper-body movement after spinal cord injury. Exp Brain Res 207:233-247. CrossRef Medline

Daffertshofer A, Lamoth CJ, Meijer OG, Beek PJ (2004) PCA in studying coordination and variability: a tutorial. Clin Biomech (Bristol, Avon) 19:415-428. CrossRef

Danziger Z, Fishbach A, Mussa-Ivaldi FA (2009) Learning algorithms for human-machine interfaces. IEEE Trans Biomed Eng 56:1502-1511. CrossRef Medline

Donders F (1848) Beitrag zur Lehre von den Bewegungen des menschlichen Auges. Holland Beit Anatom Physiolog Wiss 1:105-145.

Fischer MH, Rosenbaum DA, Vaughan J (1997) Speed and sequential effects in reaching. J Exp Psychol Hum Percept Perform 23:404-428. CrossRef Medline

Gielen CC, Vrijenhoek EJ, Flash T, Neggers SF (1997) Arm position constraints during pointing and reaching in 3-D space. J Neurophysiol 78: 660-673. Medline

Hollerbach JM, Suh K (1987) Redundancy resolution of manipulators through torque optimization. IEEE J Robot Automat 3:308-316. CrossRef

Latash ML, Scholz JP, Schöner G (2002) Motor control strategies revealed in the structure of motor variability. Exerc Sport Sci Rev 30:26-31. CrossRef Medline

Liu X, Scheidt RA (2008) Contributions of online visual feedback to the learning and generalization of novel finger coordination patterns. J Neurophysiol 99:2546-2557. CrossRef Medline

Liu X, Mosier KM, Mussa-Ivaldi FA, Casadio M, Scheidt RA (2011) Reorganization of finger coordination patterns during adaptation to rotation and scaling of a newly learned sensorimotor transformation. J Neurophysiol 105:454-473. CrossRef Medline

Medendorp WP, Crawford JD, Henriques DY, Van Gisbergen JA, Gielen CC (2000) Kinematic strategies for upper arm-forearm coordination in three dimensions. J Neurophysiol 84:2302-2316. Medline

Mosier KM, Scheidt RA, Acosta S, Mussa-Ivaldi FA (2005) Remapping hand movements in a novel geometrical environment. J Neurophysiol 94: 4362-4372. CrossRef Medline

Müller H, Sternad D (2003) A randomization method for the calculation of covariation in multiple nonlinear relations: illustrated with the example of goal-directed movements. Biol Cybern 89:22-33. Medline

Mussa-Ivaldi FA, Hogan N (1991) Integrable solutions of kinematic redundancy via impedance control. Int J Robot Res 10:481-491. CrossRef

Mussa-Ivaldi FA, Morasso P, Zaccaria R (1988) Kinematic networks. A distributed model for representing and regularizing motor redundancy. Biol Cybern 60:1-16. Medline

Park J, Zatsiorsky VM, Latash ML (2010) Optimality vs. variability: an example of multi-finger redundant tasks. Exp Brain Res 207:119-132. CrossRef Medline

Ranganathan R, Newell KM (2008) Online feedback and the regulation of degrees of freedom in motor control. Hum Mov Sci 27:577-589. CrossRef Medline

Ranganathan R, Newell KM (2009) Influence of augmented feedback on coordination strategies. J Mot Behav 41:317-330. CrossRef Medline

Rosenbaum DA, Loukopoulos LD, Meulenbroek RG, Vaughan J, Engelbrecht SE (1995) Planning reaches by evaluating stored postures. Psychol Rev 102:28-67. CrossRef Medline

Schieber MH, Santello M (2004) Hand function: peripheral and central constraints on performance. J Appl Physiol 96:2293-2300. CrossRef Medline

Scholz JP, Schöner G (1999) The uncontrolled manifold concept: identifying control variables for a functional task. Exp Brain Res 126:289-306. CrossRef Medline

Soechting JF, Buneo CA, Herrmann U, Flanders M (1995) Moving effortlessly in three dimensions: does Donders' law apply to arm movement? J Neurosci 15:6271-6280. Medline

Stevenson IH, Fernandes HL, Vilares I, Wei K, Körding KP (2009) Bayesian integration and non-linear feedback control in a full-body motor task. PLoS Comput Biol 5:e1000629. CrossRef Medline

Todorov E, Jordan MI (2002) Optimal feedback control as a theory of motor coordination. Nat Neurosci 5:1226-1235. CrossRef Medline

Vetter P, Flash T, Wolpert DM (2002) Planning movements in a simple redundant task. Curr Biol 12:488-491. CrossRef Medline 